Copyright $@ 2010$ Institute of Electrical and electronics Engineers, Inc.

All Rights reserved.

Personal use of this material, including one hard copy reproduction, is permitted.

Permission to reprint, republish and/or distribute this material in whole or in part for any other purposes must be obtained from the IEEE.

For information on obtaining permission, send an e-mail message to stds-igr@ieee.org.

By choosing to view this document, you agree to all provisions of the copyright laws protecting it.

Individual documents posted on this site may carry slightly different copyright restrictions.

For specific document information, check the copyright notice at the beginning of each document. 


\section{A Modified PSO Algorithm for Constrained Multi-Objective Optimization}

\author{
Lily D Li \\ School of Computing Sciences \\ CQUniversity \\ Rockhampton, Australia \\ l.li@cqu.edu.au \\ Xiaodong Li \\ School of Computer Science and Information \\ Technology, RMIT University \\ Melbourne, Australia \\ xiaodong@cs.rmit.edu.au
}

\begin{abstract}
This paper presents a modified PSO algorithm for solving constrained multi-objective optimization problems. Based on the constraint dominance concept, the proposed approach defines two sets of selection rules for determining the cognitive and social components of the PSO algorithm. The simulation results to the four constrained multi-objective optimization problems demonstrate the proposed approach is able to find Pareto-optimal solutions effectively.
\end{abstract}

Keywords- Constraint; Multi-objective; Optimization; PSO algorithm

\section{INTRODUCTION}

Most real-world search and optimization problems involve multiple objectives (MO) that need to be achieved simultaneously. The presence of constraints brings difficulties in optimization since the search space has to be restricted to a feasible region. In solving MO problems, three goals need to be achieved [1]: find a set of solutions as close as possible to the true Paretooptimal front; find a set of solutions as diverse as possible; and find a set of solutions as many as possible.

Population-based optimization techniques such as Genetic Algorithms (GAs), Particle Swarm Optimization (PSO), Differential Evolution (DE) and Ant Colony Optimization (ACO) have been popular choices for $\mathrm{MO}$ problems. The main reason is that these algorithms are capable of finding a set of Paretooptimal solutions in a single run. Such evolutionary optimization techniques have been extensively studied in last decade or two. Most of them are based on GAs $[1,2]$. Research shows that the PSO algorithm offers some advantages over GAs, such as simplicity and computational inexpensive $[3,4]$. With the success of

\author{
Xinghuo $\mathrm{Yu}$ \\ School of Electrical and Computer Engineering \\ RMIT University \\ Melbourne, Australia \\ x.yu@rmit.edu.au \\ William Guo \\ School of Management and Information Systems \\ CQUniversity \\ Rockhampton, Australia \\ w.guo@cqu.edu.au
}

the PSO in single objective optimization, researchers are motivated to extend the use of PSO in MO problems. Up to now, more than twenty five [5] strategies that adopt the PSO algorithm in MO problems have been proposed. Some examples are: dynamic neighbourhood [6], grid method [7], weighted aggregation [8], multi-swarm [9], dominated tree [10], fitness sharing [11], maximin strategy [12] and Huo et al [13]. A detailed review can be found in [5]. However, most of these approaches are constraint free. How to integrate constraint-handling methods with the multi-objective PSO has motivated this research.

Two papers have addressed constrained multiobjective optimization using the PSO algorithm.

Ji [14] presented a divided range multi-objective PSO for distributed computing. For constraint-handling, the author adopted the symbiosis mechanism where the feasible particles evolve towards the Pareto-front; and infeasible particles evolve toward feasibility guided by an unfeasibility function. A gradually decreased threshold is used for the proportion of infeasible particles. The approach is tested by three constrained MO functions. The author claimed that the Pareto fronts are achieved but no detailed results are given. The unfeasibility function used to guide the infeasible particles is unclear.

Reddy and Kumar [15] presented EM-MOPSO which combines the PSO technique with Pareto dominance criteria to evolve non-dominated solutions. The constraint-handling is based on the constraint dominance concept [1]. The global best particle (gBest or lBest) is randomly selected from the ERP (an external repository) where all non-dominated solutions are stored. The personal best particle is determined by the Pareto-dominance concept. The approach is tested by four constrained MO functions with the promising results. However, the approaches that use ERP will 
increase computing cost [5]. The implementation for this approach looks complicated.

In this paper, we also adopt the constraint dominance concept, and propose an easy-to-implement modified PSO algorithm for tackling constrained multiobjective optimization problems. The proposed approach defines two sets of rules for determining the cognitive and social components of the PSO algorithm. The approach has been tested by the four constrained multi-objective optimization problems and the results are presented in this paper.

The rest of the paper is organized as follows. Section II presents the proposed approach for constrained multi-objective optimization problems including the selection rules and the modified PSO algorithm. Section III presents the experiment results for the four test functions. Section IV is a performance study. Section V concludes the paper and indicates future work.

\section{THE PROPOSED APPROACH}

\section{A. Problem description}

A general multi-objective constrained optimization problem consists of a decision vector $x=\left(x_{1}, x_{2}, \ldots, x_{n}\right)$, an objective function vector $f(x)=\left(f_{1}(x), f_{2}(x), \ldots, f_{k}(x)\right)$ and a constraint function vector $g(x)=\left(g_{1}(x), g_{2}(x), \ldots, g_{m}(x)\right)$.

The problem can be stated as to find $x^{*}$ which

$$
\left.\begin{array}{lll}
\text { minimize } & f_{j}(x), & j=1,2, \ldots k \\
\text { subject to } & g_{i}(x) \leq 0, & i=1,2, \ldots m
\end{array}\right\}
$$

where $k$ is the total number of objective functions and $m$ is the total number of constraints. All solutions to a multi-objective optimization problem are called Pareto-optimal solutions, the curve by joining these solutions is known as a Pareto-optimal front.

\section{B. PSO Algorithm and Constraint Dominance Concept}

A generic PSO algorithm can be described by as follows.

$$
\begin{gathered}
v^{(t+1)}=\chi\left[w v^{(t)}+c_{1} r_{1}{ }^{(t)}\left(p B e s t^{(t)}-x^{(t)}\right)\right. \\
\left.+c_{2} r_{2}{ }^{(t)}\left(\text { lBest } t^{(t)}-x^{(t)}\right)\right] \\
x^{(t+1)}=x^{(t)}+v^{(t+1)}
\end{gathered}
$$

where $x$ is the decision vector in a D-dimensional search space; pBest is the best previous position of a particle in the flight history; lBest is the best particle of the neighbourhood; $v$ denotes the velocity; $c_{1}$ and $c_{2}$ are cognitive and social parameters respectively; $w$ is the inertia weight and $\chi$ is a constriction coefficient; $r_{l}$ and $r_{2}$ are two random numbers uniformly distributed in the range $[0,1] ; t$ denotes the iteration.

A solution's feasibility can be assessed by its total amount of constraint violations [16], described by

$$
\Phi(x)=\sum_{i=1}^{m} \max \left(0, g_{i}(x)\right)
$$

If a solution satisfies all constraints, $\Phi(x)$ returns a zero; the solution is feasible. Otherwise, $\Phi(x)$ returns a positive number; the solution is infeasible. Since an absolute equality is difficult to achieve in implementation, we use a feasibility criterion $\varepsilon$ (where $\varepsilon$ is small positive number) to evaluate a solution's feasibility, that is, a solution $\boldsymbol{x}$ is considered feasible if its $\Phi(x) \leq \varepsilon$.

The constraint dominance concept is adopted in this research. It can be described as following:

"A solution $x^{(1)}$ is said to 'constraint-dominate' a solution $x^{(2)}$, if any of the following conditions are true:

- Solution $x^{(1)}$ is feasible and solution $x^{(2)}$ is not.

- Solution $x^{(1)}$ and solution $x^{(2)}$ are both infeasible, but solution $x^{(1)}$ has a smaller constraint violation.

- Solution $x^{(1)}$ and solution $x^{(2)}$ are feasible and solution $x^{(1)}$ dominates solution $x^{(2)}$ in the usual sense" [1].

\section{Selection Rules}

Based on the constraint dominance concept, we propose the selection rules for determining pBest and lBest of the PSO algorithm, as follows.

Rule Set 1: Personal best particle updating rules

Suppose a particle's new location is pNew and its personal best location in the history is pBest:

- If both pNew and pBest are feasible, and if pNew dominates $p B e s t$, update $p B e s t$ with $p N e w$;

- If $p N e w$ is feasible and pBest is not feasible, update pBest with $p N e w$;

- If $p N e w$ is not feasible and pBest is feasible, pBest is not updated;

- If both pNew and pBest are infeasible, and if pNew has a lower constraint violations ( $\Phi$ ) than pBest has, then update $p B e s t$ with $p N e w$.

Rule Set 1 is summarized in Table I. 
TABLE I. PERSONAL BEST PARTICLE UPDATING RULES

\begin{tabular}{|l|l|l|l|l|}
\hline $\boldsymbol{p N e w}$ & $\boldsymbol{p B e s t}$ & $\begin{array}{l}\boldsymbol{p} \text { New } \\
\text { dominates } \\
\boldsymbol{p} \text { Best? }\end{array}$ & $\begin{array}{l}\text { pNew has } \\
\text { lower } \Phi \\
\text { than } \boldsymbol{p B e s t} \\
\text { has? }\end{array}$ & $\begin{array}{l}\text { Next } \\
\text { pBest }\end{array}$ \\
\hline feasible & feasible & yes & & pNew \\
\hline feasible & infeasible & & & pNew \\
\hline infeasible & feasible & & & pBest \\
\hline infeasible & infeasible & & yes & pNew \\
\hline
\end{tabular}

Rule Set 2: Local (or global) best particle selection rules:

Among a neighbourhood, select the best performed $k$ ( $k$ is number of objective functions) particles lBest $_{i}$ ( $i=1$ to $k$ ) in each objective function:

- If all $l B e s t_{i}(i=l$ to $k$ ) are feasible, randomly select one as lBest. In this way, a particle may follow lBest $_{I}$ at a time, and follow lBest $_{2}$ at another time. All lBest $_{i}(i=1$ to $k$ ) will get the same probability to be selected as lBest;

- If there exist feasible particles and infeasible particles in all lBest $_{i} \quad(i=1$ to $k$ ), the lBest is randomly selected from all the feasible particles. The infeasible particles are disregarded;

- If there are no feasible particles in all BBest $_{i}(i=1$ to $k$ ), the particle with the lowest constraint violations is selected as lBest.

For two objective optimization problems, for example, if $l B e s t_{l}$ is the best particle in objective $f_{l}$ and particle lBest $_{2}$ is the best particle in objective $f_{2}$. An arbitrary particle $p$ in the same neighbourhood will have its lBest determined by the rules listed in Table II.

TABLE II. LOCAL BEST PARTICLE SELECTION RULES

\begin{tabular}{|l|l|l|}
\hline lBest $_{\boldsymbol{1}}$ & lBest $_{\boldsymbol{2}}$ & lBest \\
\hline feasible & feasible & rand $\left(\right.$ lBest $t_{1}$, lBest $\left._{2}\right)$ \\
\hline feasible & infeasible & lBest $_{1}$ \\
\hline infeasible & feasible & lBest $_{2}$ \\
\hline infeasible & infeasible & one with the lower $\Phi$ \\
\hline
\end{tabular}

The proposed selection rules for pBest and lBest have the following features:

- The feasibility is the top priority;

- The pBest evolves towards to the bottom-left direction in objective space (assume all objectives are being minimized) since the Pareto dominance concept is adopted;

- The lBest makes effort to extend the spread along the Pareto-optimal front since the best particle in one objective function is followed at a time.

- The rules can be applied in problems that have any number of objective functions.

\section{Algorithm}

Table III is the structure of the modified PSO algorithm for constrained multi-objective optimization problems.

TABLE III. STRUCTURE OF THE MODIFIED PSO ALGORITHM

\footnotetext{
Initialize particles

Calculate fitness values of particles under each objective

Calculate constraint violations of each particle

Set current locations as personal best locations

Set local best location for each particle according to Rule Set 2

Do

For each particle

Calculate new velocity by PSO formula

Calculate new location by PSO formula

Update personal best location according to Rule Set 1

End For

Set local best location for each particle according to Rule Set 2 End Do
}

\section{EXPERIMENTS}

\section{A. Test Functions}

Four functions (taken from [1]), named BNH, TNK, SRN and OSY have been selected for testing the proposed approach. The $\mathrm{BNH}$ function has a continuous convex Pareto-optimal set. The TNK function has a discontinuous, convex and nonconvex Pareto-optimal set. Both the SRN function and the OSY function have continuous linear (can be considered convex or nonconvex) Pareto-optimal sets. The TNK and the OSY functions are considered difficult problems since the TNK function has a discontinuous Pareto-front and the OSY function is a high dimensional and a highly constrained problem. The functions are described in equations (5) to (8).

BNH:

$$
\begin{cases}\text { Minimize } & f_{1}(\mathbf{x})=4 x_{1}{ }^{2}+4 x_{2}{ }^{2}, \\ & f_{2}(\mathbf{x})=\left(x_{1}-5\right)^{2}+\left(x_{2}-5\right)^{2}, \\ \text { subject to } & g_{1}(\mathbf{x})=\left(x_{1}-5\right)^{2}+x_{2}{ }^{2}-25 \leq 0, \\ & g_{2}(\mathbf{x})=7.7-\left(x_{1}-8\right)^{2}-\left(x_{2}+3\right)^{2} \leq 0, \\ & 0 \leq x_{1} \leq 5, \quad 0 \leq x_{2} \leq 3 .\end{cases}
$$

TNK:

$$
\begin{cases}\text { Minimize } & f_{1}(\mathbf{x})=x_{1}, \\ & f_{2}(\mathbf{x})=x_{2}, \\ \text { subject to } & g_{1}(\mathbf{x})=1-x_{1}^{2}-x_{2}^{2}+0.1 \cos \left(16 \arctan \frac{x_{1}}{x_{2}}\right) \leq 0, \\ & g_{2}(\mathbf{x})=\left(x_{1}-0.5\right)^{2}+\left(x_{2}-0.5\right)^{2}-0.5 \leq 0, \\ & 0 \leq x_{1}, x_{2} \leq \pi .\end{cases}
$$


SRN:

$$
\begin{cases}\text { Minimize } & f_{1}(\mathbf{x})=2+\left(x_{1}-2\right)^{2}+\left(x_{2}-1\right)^{2}, \\ & f_{2}(\mathbf{x})=9 x_{1}-\left(x_{2}-1\right)^{2} \\ \text { subject to } & g_{1}(\mathbf{x})=x_{1}{ }^{2}+x_{2}^{2}-225 \leq 0 \\ & g_{2}(\mathbf{x})=x_{1}-3 x_{2}+10 \leq 0 \\ & -20 \leq x_{1}, x_{2} \leq 20\end{cases}
$$

OSY:

$$
\left\{\begin{aligned}
\text { Minimize } \quad & f_{1}(\mathbf{x})=-\left[25\left(x_{1}-2\right)^{2}+\left(x_{2}-2\right)^{2}+\left(x_{3}-1\right)^{2}\right. \\
& \left.+\left(x_{4}-4\right)^{2}+\left(x_{5}-1\right)^{2}\right], \\
& f_{2}(\mathbf{x})=x_{1}^{2}+x_{2}^{2}+x_{3}^{2}+x_{4}^{2}+x_{5}^{2}+x_{6}^{2}, \\
\text { subject to } \quad & g_{1}(\mathbf{x})=2-x_{1}-x_{2} \leq 0, \\
& g_{2}(\mathbf{x})=-6+x_{1}+x_{2} \leq 0, \\
& g_{3}(\mathbf{x})=-2-x_{1}+x_{2} \leq 0, \\
& g_{4}(\mathbf{x})=-2+x_{1}-3 x_{2} \leq 0, \\
& g_{5}(\mathbf{x})=-4+\left(x_{3}-3\right)^{2}+x_{4} \leq 0, \\
& g_{6}(\mathbf{x})=4-\left(x_{5}-3\right)^{2}-x_{6} \leq 0, \\
& 0 \leq x_{1}, x_{2}, x_{6} \leq 10, \quad 1 \leq x_{3}, x_{5} \leq 5, \quad 0 \leq x_{4} \leq 6 .
\end{aligned}\right.
$$

\section{B. Results}

For multi-objective optimization, the number of non-dominated solutions is directly linked to the population size. Therefore, a large size of population is required.

Twenty independent runs have been performed for each case. The PSO parameters are: $c_{1}=1.0$; $c_{2}=2.0 ; V_{\max }=0.5 \cdot(U-L)$, where $U$ and $L$ are the upper and lower boundary for the decision variables; the population size is 200 for the BNH and the SRN functions; the population size is 500 for the TNK and the OSY functions; the maximum iteration $i_{\max }$ for all four cases is 1000; the inertia weight $w=0.1$; $\chi=0.63$; a potential solution is considered feasible when its $\Phi<\varepsilon=1.0 E-05$; Neighbourhood topology is set to the circular ring local model with the neighbourhood size two.

Figures 1 to 4 illustrate the simulated Pareto-optimal fronts from the best runs for the four test cases. The best run means that the Pareto-optimal front has a small Spacing/Spread (S/D) value. 156 out of 200 nondominated solutions are found for the BHN function. 111 out of 500 non-dominated solutions are found for the TNK function. 161 out of 200 non-dominated solutions are found for the SRN function and 56 out of 500 non-dominated solutions are found for the OSY function. The algorithm achieved reasonably good number of non-dominated solutions for the first three functions. However, the number of non-dominated solutions found for the last function (OSY) is small due to the complexity of the problem.
By observing the simulated Pareto-optimal fronts and the theoretical Pareto-optimal fronts [1], the proposed approach is able to converge to the Paretooptimal solutions effectively. The final solution curves are reasonably dispersed.

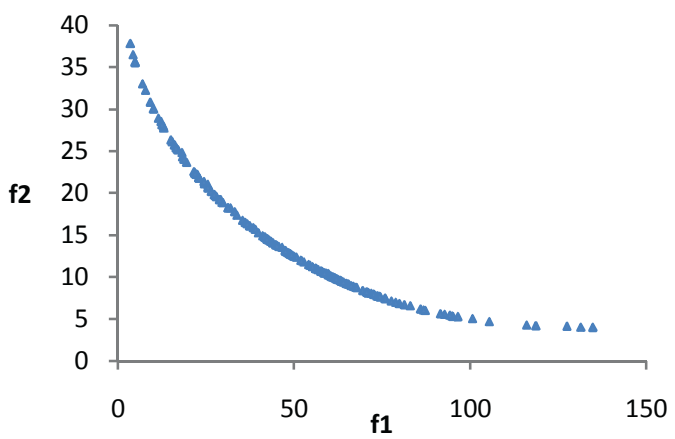

Figure 1. Simulated Pareto-optimal front for test problem $\mathrm{BNH}$

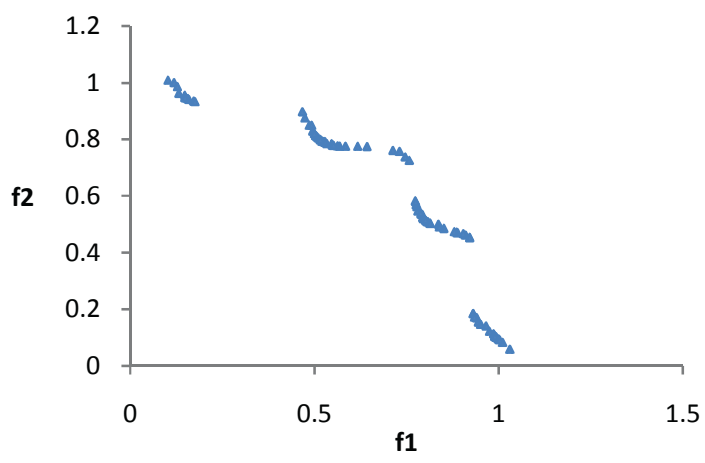

Figure 2. Simulated Pareto-optimal front for test problem TNK

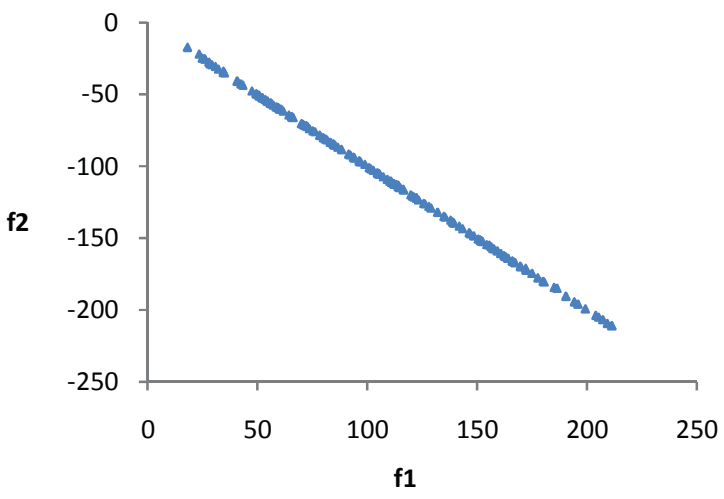

Figure 3. Simulated Pareto-optimal front for test problem SRN 


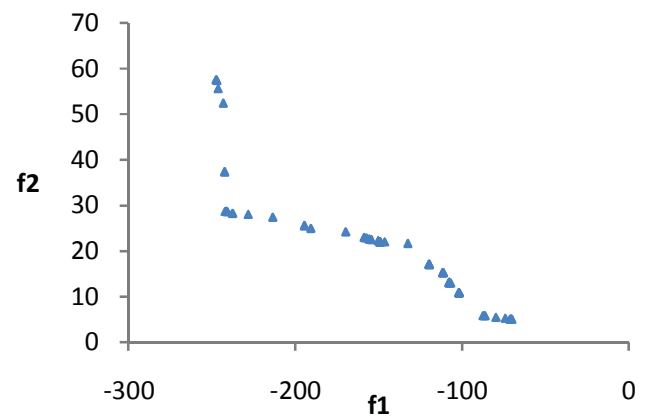

Figure 4. Simulated Pareto-optimal front for test problem OSY

\section{PERFORMANCE STUDY}

Experiments are conducted based on the two metrics, "Spacing" and "Maximum Spread" (details in Appendix A). Table IV presents the results for the four functions. Since a smaller "Spacing" (S) value and a larger "Spread" (D) value are desirable, the minimum S and the maximum $\mathrm{D}$ are listed in the table. The average values and the standard deviations are also included.

The "Generational Distance" is not evaluated at this stage because of the lack of the real Pareto front data.

Due to the complexity and different user demands, there is not much data available for performance comparison. We include the "Spacing" data (EMMOPSO) from [15] in Table V for a brief comparison. No "Max Spread" data available from [15]. The NSGAII data in Table $\mathrm{V}$ is also taken from [15].

TABLE IV. SPACING AND MAXIMUM SPREAD FOR TESTING CASES BASED ON 20 RUNS

\begin{tabular}{|l|l|l|l|r|r|}
\hline & Item & BNH & TNK & SRN & OSY \\
\hline \multirow{4}{*}{ S } & Min. & 0.599672 & 0.004032 & 1.089612 & 1.040084 \\
\cline { 2 - 6 } & Avg. & 0.979688 & 0.012609 & 1.587708 & 2.943298 \\
\cline { 2 - 6 } & Std. & 0.258917 & 0.003361 & 0.423624 & 1.057702 \\
\hline \multirow{3}{*}{ D } & Max. & 143.5117 & 1.403277 & 302.6255 & 225.3124 \\
\cline { 2 - 6 } & Avg. & 136.9071 & 1.362672 & 276.0655 & 155.4652 \\
\cline { 2 - 6 } & Std. & 3.775605 & 0.031040 & 16.26418 & 21.64976 \\
\hline
\end{tabular}

TABLE V. STATISTIC RESULTS BY DIFFERENT APPROACHES

\begin{tabular}{|l|l|l|l|l|}
\hline & & This Paper & NSGA-II & EM-MOPSO \\
\hline \multirow{4}{*}{ BNH } & Min. & 0.599672 & 0.6408 & 0.6357 \\
\cline { 2 - 5 } & Avg. & 0.979688 & 0.7756 & 0.6941 \\
\cline { 2 - 5 } & Std. & 0.258917 & 0.0727 & 0.0385 \\
\hline \multirow{3}{*}{ SRN } & Min. & 1.089612 & 1.3402 & 1.0768 \\
\cline { 2 - 5 } & Avg. & 1.587708 & 1.5860 & 1.2439 \\
\cline { 2 - 5 } & Std. & 0.423624 & 0.1337 & 0.1055 \\
\hline
\end{tabular}

According to Table V, our approach obtained the best "Spacing" value in function BNH. For function
SRN, our approach outperformed NSGA-II in the best "Spacing" value but slightly worse than EM-MOPSO. Regarding to algorithm consistency, our approach did not outperform the other two approaches in both functions but was in the acceptable range. More investigation needs to be done about this.

We realize that the "Spacing" value can only be applied in consecutive solutions which may not be suitable for those problems that have discontinuous solutions such as function TNK. In such cases, other performance metrics may be taken into consideration.

\section{CONCLUSIONS AND FUTURE WORK}

This paper proposed a simple and easy-toimplement modified PSO algorithm for solving constrained multi-objective optimization problems. By adopting the constraint dominance concept, the proposed approach defines two sets of rules for determining the personal best location pBest and the best neighbourhood location lBest of the PSO algorithm. The simulation results to the four constrained multi-objective optimization problems demonstrated the proposed approach is able to find the Pareto-optimal solutions effectively.

The performance of the proposed approach has been evaluated by two metrics. The results demonstrated the proposed approach is able to achieve similar good results in best "spacing" to the compared approaches. However, the proposed approach did not outperform the compared approaches in terms of consistency. Our future work includes more investigation in diversity control and testing the proposed approach in problems that have more than two objective functions.

\section{APPENDIX A}

Three metrics can be used for performance evaluation, as follows.

Spacing (S) [17] measures how well distributed (spaced) the solutions in the non-dominated set found. The formula is presented in (9),

$$
S=\sqrt{\frac{1}{n} \sum_{i=1}^{n}\left(d_{i}-\bar{d}\right)^{2}}
$$

where $n$ is the number of solutions in the obtained non-dominated set, $d_{i}=\min _{k \in n \wedge k \neq i} \sum_{m=1}^{M}\left|f_{m}{ }^{i}-f_{m}{ }^{k}\right|$ and $\bar{d}$ is the mean value of the above distance measure $\bar{d}=\sum_{i=1}^{n} d_{i} / n ; f_{m}$ is the $m$-th objective function value; $M$ is the number of objective functions. When the solutions are near uniformly spaced, the corresponding 
distance measure will be small. Thus, an algorithm finding a set of non-dominated solutions having a smaller spacing $S$ is better.

Maximum Spread [18] gives a value which represents the maximum extension between the farthest solutions in the non-dominated set found. The formula is presented in (10). A bigger value indicates better performance.

$$
D=\sqrt{\sum_{m=1}^{M}\left(\max _{i=1}^{n} f_{m}{ }^{i}-\min _{i=1}^{n} f_{m}{ }^{i}\right)^{2}}
$$

Generational Distance (as cited in [1]) is a metric to find the average distance of the non-dominated set of solutions from the real Pareto optimal set. The formula is given in (11).

$$
G D=\frac{\sqrt{\sum_{i=1}^{n} d_{i}{ }^{2}}}{n}
$$

where $d_{i}$ is the Euclidean distance between solution $i$ from the set of $n$ non-dominated solutions found and the closest element from the real Pareto optimal set. A smaller value indicates the solutions found are closer to the real Pareto front.

\section{REFERENCES}

[1] K. Deb, Multi-Objective Optimization using Evolutionary Algorithms: John Wiley \& Sons, 2001.

[2] Ruhul Sarker, Masoud Mohammadian, and X. Yao, "Evolutionary Optimization," in International Series in Operations Research \& Management Science, F. S. Hillier, Ed., 2002.

[3] J. Kennedy, R. Eberhart, and Y. Shi, Swarm Intelligence: San Francisco: Morgan Kaufmann Publisher, 2001.

[4] K. E. Parsopoulos and M. N. Vrahatis, "Recent Approaches to Global Optimization Problems Through Particle Swarm Optimization," Natural Computing, vol. 1, pp. 235-306, 2002.

[5] M. Reyes-Sierra and C. A. C. Coello, "Multi-Objective Particle Swarm Optimizers: A Survey of the State-of-the-Art," International Journal of Computational Intelligence Research, vol. 2, pp. 287-308, 2006.

[6] X. Hu and R. Eberhart, "Multiobjective Optimization Using Dynamic Neighborhood Particle Swarm Optimization," in
Proceeding of IEEE Congress on Evolutionary Computation, Honolulu, Hawaii, 2002.

[7] C. A. C. Coello and M. S. Lechuga, "MOPSO: A Proposal for Multiple Objective Particle Swarm Optimization," in Proceedings of IEEE Congress on Evolutionary Computation. vol. 2 Honolulu, HI, USA, 2002, pp. 1051-1056.

[8] K. E. Parsopoulos and M. N. Vrahatis, "Particle Swarm Optimization Method in Multiobjective Problems," in Proceeding of ACM Symposium on Applied Computing Madrid, Spain, 2002, pp. 603-607.

[9] K. E. Parsopoulos and M. N. Vrahatis, "Multiobjective Optimization using Parallel Vector Evaluated Particle Swarm Optimization," in In Proceedings of the IASTED International Conference on Artificial Intelligence and Applications,: ACTA Press, 2004, pp. 823--828.

[10] J. E. Fieldsend and S. Singh, "A Multi-objective Algorithm Based upon Particle Swarm Optimization, An Efficient Data Structure and Turbulence," in Proceeding of UK Workshop on Computational Intelligence (UKCI'02), Birminghan, UK, 2002, pp. 37-44.

[11] M. Salazar-Lechuga and J. E. Rowe, "Particle Swarm Optimization and Fitness Sharing to solve Multi-Objective Optimization Problems," in Proceedings of IEEE Congress on Evolutionary Computation, Edinburgh, UK, 2005, pp. 12041211.

[12] X. Li, "Better Spread and Convergence: Particle Swarm Multiobjective Optimization Using the Maximin Fitness Function," in Proceedings of the Genetic and Evolutioanry Computation Conference (GECCO'2004) Seattle, Washington, USA: Springer-Verlag, Lecture Notes in Computer Science Vol.3102, 2004, pp. 117-128.

[13] X. Huo, L. Shen, and H. Zhu, "A Smart Particle Swarm Optimization Algorithm for Multi-Objective Problems," in Lecture Notes in Computer Science. vol. 4115/2006, 2006.

[14] C. Ji, "A Revised Particle Swarm Optimization Approach for Multi-objective and Multi-constraint Optimization," in Proceeding of 2004 Genetic and Evolutionary Computation Conference (GECCO) Seattle, Washington, USA, 2004.

[15] M. J. Reddy and D. N. Kumar, "Multi-objective Particle Swarm Optimization for Generating Optimal Trade-offs in Reservoir Operation," Hydrological Process, vol. 21, pp. 2897-2909, 2007

[16] E. Camponogara and S. N. Talukdar, "A Genetic Algorithm For Constrained and Multiobjective optimization," in Proceeding of 3rd Nordic Workshop on Genetic Algorithms and Their Applications, Vaasa, Finland, 1995, pp. 49-62.

[17] J. R. Schott, Fault Tolerant Design Using Single and MultiCriteria Genetic Algorithms vol. Masters Thesis: Massachusetts Institute of Technology, 1995.

[18] E. Zitzler and L. Thiele, "Multiobjective Evolutionary Algorithms: A Comparative Case Study and the Strength Pareto Approach," IEEE TRANSACTIONS ON EVOLUTIONARY COMPUTATION,, vol. 3 (4), pp. 257-271, 1999. 